\title{
Computing Cournot Equilibrium through Maximization over Prices*
}

\author{
Hakan Orbay ${ }^{\dagger}$ \\ Sabanc1 University
}

August 2007

\begin{abstract}
This paper presents an alternative characterization of internal Cournot equilibrium based on the first-order conditions corresponding to profit maximization over prices. This characterization is particularly useful when the market is described in terms of demand functions (rather than inverse-demand functions). A significant computational advantage is gained in homogeneous good cases as demand functions need not be inverted and simple first order conditions are obtained. In addition, this approach mimics price setting behavior and thus suggests a price competition interpretation of the Cournot game.
\end{abstract}

${ }^{*}$ This paper originated during our joint work with Benan Orbay on a related paper, for which I am grateful to her. I would also like to thank Ozgur Kibris, Kieron Meagher and Murat Usman for their comments and suggestions on earlier versions.

†Sabancı University, Orhanli, Tuzla 34956 Istanbul, Turkey; e-mail: horbay@sabanciuniv.edu. 


\section{Introduction}

The usual approach to computing the Cournot equilibrium consists of determining inverse demand functions and expressing the profit functions of the firms as a function of quantity choices. When a certain situation is modeled starting with consumer choices, it is typical that the market is described with a demand function, which needs to be inverted to find the Cournot equilibrium. There are, however, cases of interest for which inverse demand functions cannot be determined in an analytical form, and consequently, direct computation of the Cournot equilibrium is not feasible. The sole purpose of this paper is to point out that Cournot equilibrium can also be determined by using prices as instruments to maximize profit functions. This approach obviates the need for inverting demand functions while yielding a simpler system of equations compared to the direct approach; thus, the proposed method is appealing whenever the market is described in terms of demand functions.

In the case of purely differentiated goods, the equivalence of using quantities or prices as maximizing instruments is clear, as can be seen in Vives (1985). What is less appreciated is that, the equivalence also holds for the homogeneous goods case, provided that there are no corner solutions in the maximization problems. I will start with a simple example in order to demonstrate equivalence of using prices as instruments.

Example 1. Consider the standard one-good, two-firm model with linear demand, $Q=1-p$, and constant marginal costs $c^{1}$ and $c^{2}$ for firms 1 and 2, both less than 1. Let firm 1 maximize its profit function, $\pi^{1}=\left(p-c^{1}\right) q^{1}$, over $p$. The first-order condition $\frac{d \pi^{1}}{d p}=0$ yields,

$$
q^{1}+\left(p-c^{1}\right) \frac{d q^{1}}{d p}=0
$$

Note that $q^{1}=Q-q^{2}$. In the Cournot-Nash paradigm where quantities are strategic variables, (1) defines best response function of Firm 1, given Firm 2's choice of quantity. Hence, $q^{2}$ must be taken as fixed within (1), or $\frac{d q^{2}}{d p}=0 .{ }^{1}$ Thus, $\frac{d q^{1}}{d p}=\frac{d Q}{d p}=-1$. Together with the same for Firm 2, we have the following two first order conditions,

$$
\begin{aligned}
& q^{1}=p-c^{1} \\
& q^{2}=p-c^{2}
\end{aligned}
$$

Adding the two conditions above gives the standard Cournot equilibrium with $p=\left(1+c^{1}+c^{2}\right) / 3$.

\footnotetext{
${ }^{1}$ Admittedly, this is an abuse of notation, which is adopted to simplify exposition. To be precise, $p$ should be replaced with $p^{1}$, a dummy variable referring to price set by Firm 1 . Then, any equilibrium requires that equality of both prices.
} 
The example above demonstrates computation of Cournot equilibrium thorough maximization over prices. The following example shows why this approach may be crucial for analysis.

Example 2. Consider a market with two firms and three homogeneous goods with the following demand functions:

$$
\begin{aligned}
& Q_{1}=\left(1-p_{1}\right)\left(-p_{1}+p_{3}\right) \\
& Q_{2}=\left(1-p_{2}\right)\left(-p_{2}+p_{3}\right) \\
& Q_{3}=1+p_{1}+p_{2}-2 p_{3}+\frac{p_{3}^{2}-p_{1}{ }^{2}-p_{2}{ }^{2}}{2}
\end{aligned}
$$

These demand functions cannot be inverted analytically, hence direct calculation of the Cournot equilibrium is quite difficult. However, through maximization over prices, immediate analytical solutions can be obtained.

Let me note that the demand functions in Example 2 arises where Good 3 is a bundle of Good 1 and Good 2, and consumer valuations in unbundled goods are are i.i.d. with uniform distribution over $[0,1]$. This setup appears in a number of papers regarding strategic bundling behavior, such as Nalebuff (1999), which assumes that one firm is a monopoly in both goods, but faces Bertrand competition with a potential entrant in only one good. Indeed, Cournot equilibrium is markedly absent in this literature; models usually adopt markets with differentiated products and consider Bertrand equilibrium, or assume absence of strategic behavior in one of the goods. ${ }^{2}$

I will proceed by an informal proof of the proposed method, which is essentially an alternative characterization of the Cournot equilibrium. Taking example 2, suppose that there is a Cournot euilibrium where both firms produce all 3 goods. In equilibrium, neither firm has an incentive to deviate from the equilibrium by changing the quantity of a single good. The direct method proceeds by specifying these deviations one good at a time. However, it is equally true that neither firm has an incentive to deviate by changing its output of several goods at once. For example, increasing production of good 1 by $x$ and decreasing production of good 2 by $2 x$ cannot be profitable if changing the production of each good alone is not profitable. This observation relies on the simple fact that all directional derivatives are zero at the optimum, and a deviation involving multiple goods is a movement in an arbitrary direction.

Consequently, another set of first-order conditions may be written where each equation considers a deviation with multiple goods and sets 3 directional derivatives to zero. This is equivalent to the first-order conditions obtained from the direct method, provided that the 3 directions are independent. In particular, these directions may be chosen in such a manner that, as a firm

\footnotetext{
${ }^{2}$ For example, Carbajo, Meza \& Seidmann (1990), Whinston (1990) and Martin (1999) consider models where one firm is a monopolist in one of the goods. In Chen (1997), one of the goods have a perfectly competitive market. Matutes \& Regibeau (1992) and Anderson \& Leruth (1993) are among the few that consider duopolistic competition in two products. Both of the latter employ Bertrand competition and assume that the goods are perfect complements. The detailed analysis of the Cournot equilibria of this model is taken up in Orbay \& Orbay (2006).
} 
changes its output along each direction, only one market-clearing price changes and all other prices remain constant. The original problem is thus converted into one where market prices appear to be the strategic choice variables. That is, each first-order condition is equivalent to requiring that a firm has no incentive to change the market price of one good. The Cournot paradigm is preserved since a firm can only change a price by changing its outputs, and the other firms' quantities are taken to be fixed. The new set of first order conditions require differentiation of the demand functions w.r.t. to prices only.

As can be deduced from the discussion above, maximization over prices method can only be used if all directional derivatives are zero at the optimum. A sufficient (but not necessary) condition to satisfy this requirement is that profit functions are continuously differentiable and all firms produce strictly positive amounts of each good.

Computational simplification suggested in the above discussion is achieved only if every firm produces all the goods in equilibrium. For this reason, main exposition is restricted to internal equilibria of the homogenous-good case in section 2. Section 3 elaborates on the relationship between Bertrand and Cournot equilibria in a differentiated good setting.

\section{Cournot Equilibrium in Homogeneous Goods}

\subsection{Definitions and Notation}

Consider a market where $n$ firms produce $m$ goods. Let $\mathbf{Q} \in \Re_{+}^{m}$ be the total quantities of $m$ goods, and $\mathbf{p} \in \Re_{+}^{m}$ denote the market prices for those goods. The demand for the goods are given with the demand function

$$
\mathbf{Q}=\mathbf{f}(\mathbf{p})
$$

where $\mathbf{f}: \Re_{+}^{m} \rightarrow \Re_{+}^{m}$ is continuous and twice differentiable. The function f is interpreted as the collection of demand functions for individual goods, $Q_{j}=f_{j}\left(p_{1}, \ldots, p_{m}\right)$, where the subscripts refer to a particular good. The firms compete in this market by choosing quantities supplied for each good. Let $\mathbf{Q}^{i} \in \Re_{+}^{m}$ denote the production choice (strategy) of firm $i$. Firm $i$ maximizes its profit function,

$$
\Pi^{i}\left(\mathbf{Q}^{i}, \mathbf{p}\right)=\mathbf{p}^{\mathbf{T}} \cdot \mathbf{Q}^{i}-c^{i}\left(\mathbf{Q}^{i}\right) .
$$

where $c^{i}\left(\mathbf{Q}^{i}: \Re_{+}^{m} \rightarrow \Re\right.$ represents total cost production and is continuously differentiable. Assume that $\Pi^{i}$, for all $i$, has appropriate concavity conditions such that the first-order conditions are sufficient to determine the unique optima of the $n$ profit functions. Sufficient conditions are that $\mathbf{p}^{\mathbf{T}} \cdot \mathbf{f}(\mathbf{p})$ is quasi-concave and $c^{i}$ are convex, differentiable functions. ${ }^{3}$

Definition 1 The collection of outputs and market prices, $\left\langle\mathbf{Q}^{1 *}, \ldots, \mathbf{Q}^{n *}, \mathbf{p}^{*}\right\rangle$ is a Cournot equilibrium of the market if each firm's output maximizes its profit

\footnotetext{
${ }^{3}$ For details regarding the existence and uniqueness of Cournot equilibrium see, for example, Tirole (1988, p. 224) and the references therein.
} 
function given the outputs of the other firms and market clears, i.e.,

$$
\sum_{i=1}^{m} \mathbf{Q}^{i}=\mathbf{f}(\mathbf{p})
$$

This general definition of the Cournot equilibrium does not exclude the cases where the profit is maximized at a boundary (such as, zero output of a good by a firm) where a first-order condition is violated. For our purposes, we will adopt the following definition in order to ensure the validity of the characterization by first-order conditions.

Definition 2 A Cournot equilibrium is called an internal equilibrium if $\left|\mathbf{D f}\left(\mathbf{p}^{*}\right)\right| \neq$ 0 and the first-order conditions for profit maximization is satisfied for all firms, i.e.,

$$
\mathbf{D}_{\mathbf{Q}^{i}} \Pi^{i}\left(\mathbf{Q}^{i *}, \mathbf{f}^{-1}\left(\sum_{k=1}^{n} \mathbf{Q}^{k *}\right)\right)=\mathbf{0} \quad i=1, \ldots, n
$$

where $\mathbf{f}^{-1}$ is the inverse-demand function. ${ }^{4}$

Note that, a necessary condition for the existence of the Cournot equilibrium is that there must exist market clearing prices for the equilibrium quantities supplied. From the implicit function theorem, such prices exist if and only if the Jacobian determinant of the demand function $\mathbf{f}$ is non-zero at the equilibrium, $\left|\mathbf{D f}\left(\mathbf{p}^{*}\right)\right| \neq 0$, which ensures that $\mathbf{f}^{-1}\left(\mathbf{Q}^{*}\right)$ exists.

\subsection{Characterizing Internal Cournot Equilibrium via Prices}

The following theorem shows that internal Cournot equilibrium can be characterized through first-order conditions derived from maximization over prices.

Theorem $1\left\langle\mathbf{Q}^{1 *}, \ldots, \mathbf{Q}^{n *}, \mathbf{p}^{*}\right\rangle$ is an internal Cournot equilibrium if and only if $\left|\mathbf{D f}\left(\mathbf{p}^{*}\right)\right| \neq 0$ and satisfies,

$$
\mathbf{D}_{\mathbf{p}} \Pi^{i}\left(\mathbf{Q}^{i}\left(\mathbf{p}^{*}\right), \mathbf{p}^{*}\right)=\mathbf{0} \quad i=1, \ldots, n
$$

where $\mathbf{D Q}^{i}\left(\mathbf{p}^{*}\right):=\mathbf{D} \mathbf{f}\left(\mathbf{p}^{*}\right) .{ }^{5}$

Proof. In an internal equilibrium the first-order conditions (7) must hold, which can be written as

$$
\mathbf{D}_{\mathbf{Q}^{i}} \Pi^{i}\left(\mathbf{Q}^{i *}, \mathbf{p}^{*}\right)+\mathbf{D}_{\mathbf{p}} \Pi^{i}\left(\mathbf{Q}^{i *}, \mathbf{p}^{*}\right) \mathbf{D}_{\mathbf{Q}^{i}} \mathbf{f}^{-1}\left(\mathbf{Q}^{*}\right)=\mathbf{0} \quad i=1, \ldots, n .
$$

\footnotetext{
${ }^{4}$ Throughout the paper, $\mathbf{D}$ is used as the differentiation operator. Hence, if $\mathbf{g}: \Re^{k} \rightarrow \Re^{l}$, $\mathbf{D g}(\mathbf{x})$ is the $l \times k$ Jacobian matrix of $\mathbf{g}$ at $\mathbf{x}$. Furthermore, if $\mathbf{y} \in \Re^{j}, \mathbf{D}_{\mathbf{y}} \mathbf{g}$ denotes the partial differentiation matrix of $\mathbf{g}$ w.r.t. $\mathbf{y}$, which has dimensions $l \times j$.

${ }^{5}$ The assignment operator $:=$ should be read as 'is defined to be'.
} 
Note that since $\mathbf{D}_{\mathbf{Q}^{i}} \mathbf{Q}=\mathcal{I}_{m}$ (identity matrix), $\mathbf{D}_{\mathbf{Q}^{i}} \mathbf{f}^{-1}\left(\mathbf{Q}^{*}\right)=\mathbf{D}_{\mathbf{Q}} \mathbf{f}^{-1}\left(\mathbf{Q}^{*}\right)$. Furthermore, $\mathbf{D}_{\mathbf{Q}} \mathbf{f}^{-1}\left(\mathbf{Q}^{*}\right)=\left(\mathbf{D} \mathbf{f}\left(\mathbf{p}^{*}\right)\right)^{-1}$ from the implicit function theorem, given that $\left|\mathbf{D f}\left(\mathbf{p}^{*}\right)\right| \neq 0$. Thus, post-multiplying above equalities with $\mathbf{D f}\left(\mathbf{p}^{*}\right)$ yields

$$
\mathbf{D}_{\mathbf{Q}^{i} \Pi^{i}}\left(\mathbf{Q}^{i *}, \mathbf{p}^{*}\right) \mathbf{D f}\left(\mathbf{p}^{*}\right)+\mathbf{D}_{\mathbf{p}} \Pi^{i}\left(\mathbf{Q}^{i *}, \mathbf{p}^{*}\right)=\mathbf{0} \quad i=1, \ldots, n,
$$

which is equivalent to (8) given that $\mathbf{D}_{\mathbf{p}} \mathbf{Q}^{i}\left(\mathbf{p}^{*}\right)$ is set to be $\mathbf{D f}\left(\mathbf{p}^{*}\right)$.

The proof essentially shows the equivalence of the two maximization problems, $\max _{q_{1}^{i} \ldots, q_{m}^{i}} \Pi^{i}$ and $\max _{p_{1}, \ldots, p_{m}} \Pi^{i}$, when the demand functions are invertible and the first-order conditions are satisfied at the optimum. The message of this characterization is that an internal Cournot equilibrium can be computed by letting the firms use market prices as if they are the strategic variables.

Cournot paradigm is preserved through the statement $\mathbf{D}_{\mathbf{p}} \mathbf{Q}^{i}\left(\mathbf{p}^{*}\right):=\mathbf{D f}\left(\mathbf{p}^{*}\right)$. Given that $\mathbf{f}(\mathbf{p})=\sum_{k=1}^{n} \mathbf{Q}^{k}$, this statement is equivalent to holding quantities of all other firms constant. This does not apply to the Bertrand equilibrium; indeed $\mathbf{D}_{\mathbf{p}} \mathbf{Q}^{i}\left(\mathbf{p}^{*}\right)$ is either zero or unbounded under price competition.

Corollary 2 Any solution to the following system of $n \times m$ equations,

$$
\left(\mathbf{Q}^{i}\right)^{\mathbf{T}}=\left(\mathbf{p}^{\mathbf{T}}-\mathbf{D} c^{i}\left(\mathbf{Q}^{i}\right)\right) \mathbf{D f}(\mathbf{p}) \quad i=1, \ldots, n
$$

is a Cournot equilibrium of the market provided that $|\mathbf{D f}(\mathbf{p})| \neq 0$ at the solution.

Proof. From (6), $\mathbf{D}_{\mathbf{Q}^{i}} \Pi^{i}\left(\mathbf{Q}^{i}, \mathbf{p}\right)=\mathbf{p}^{\mathbf{T}}-\mathbf{D} c^{i}\left(\mathbf{Q}^{i}\right)$ and $\mathbf{D}_{\mathbf{p}} \Pi^{i}\left(\mathbf{Q}^{i}, \mathbf{p}\right)=\left(\mathbf{Q}^{i}\right)^{\mathbf{T}}=$ $\mathbf{Q}^{\mathbf{T}} \mathbf{s}^{i}$. Thus, the corollary follows from Theorem 1 .

Using (9), the Cournot equilibrium of the market can be determined without any need for inverting the demand functions, and may yield significant computational advantage as demonstrated below. For firm $i$ and good $j,(9)$ can be written as,

$$
q_{j}^{i}=-\sum_{k=1}^{m}\left(p_{k}-\mathbf{D}_{q_{k}} c\left(\mathbf{Q}^{i}\right)\right) \mathbf{D}_{p_{j}} f_{k}(\mathbf{p}),
$$

which is no more than equating marginal revenue and marginal loss of the market prices.

The following well-known property, which follows immediately from Corollary 2 , demonstrates the simplicity of the first-order conditions in (9).

Corollary $\mathbf{3}$ In any internal Cournot equilibrium, $\mathbf{Q}^{i}=\mathbf{Q}^{j}$ iff $\mathbf{D} c^{i}\left(\mathbf{Q}^{i}\right)=$ $\mathbf{D} c^{j}\left(\mathbf{Q}^{j}\right)$. Moreover, $\mathbf{D} c^{i}\left(\mathbf{Q}^{i}\right)>\mathbf{D} c^{j}\left(\mathbf{Q}^{j}\right) \Rightarrow \mathbf{Q}^{i}<\mathbf{Q}^{j}$.

Example 2 revisited. I would like to demonstrate the computational simplicity afforded by the proposed method through the mixed-bundling problem introduced in Example 2 above. The demand functions are given in (4), but it should be noted that these are subject to the constraints $p_{1}, p_{2} \in[0,1], p_{1}, p_{2} \leq p_{b}$ and $p_{b} \leq p_{1}+p_{2}$, as good 3 is a bundle of goods 1 and 2 . In addition, assume that both firms have zero marginal costs for simplicity. 
Let us start with the supposition that there is an internal Cournot equilibrium. By Corollary 3, both firms have equal quantities of all goods, thus $\mathbf{Q}^{1}=\mathbf{Q}^{2}=\mathbf{Q} / 2$. With zero marginal costs, (9) reduces to,

$$
Q_{j} / 2=-\sum_{k=1}^{3} p_{k} \frac{\partial Q_{k}}{\partial p_{j}} \quad j=1,2,3
$$

Computing (11) and simplifying yields the following equations:

$$
\begin{aligned}
\left(-3+5 p_{1}\right)\left(p_{1}-p_{3}\right) & =0 \\
\left(-3+5 p_{2}\right)\left(p_{2}-p_{3}\right) & =0 \\
2+6 p_{1}-5 p_{1}{ }^{2}+6 p_{2}-5{p_{2}}^{2}-12 p_{3}+5 p_{3}{ }^{2} & =0
\end{aligned}
$$

The first two equations give $p_{1}=p_{2}=0.6$. Solving the remaining equation with these values yield $p_{3}=(6-2 \sqrt{2}) / 5$. As these prices are feasible, this is a Cournot equilibrium. ${ }^{6}$

The first-order conditions w.r.t. prices can be written for a firm only if that firm is able to vary each price alone. In general, this requires that the firm should be able to change its output of all the goods in the market; that is why the restriction to internal equilibria is required. In a Cournot equilibrium that is not internal, (9) would not be applicable as at least a firm will be constrained in some manner. As an example, consider a model with two firms and two goods, where firm 2 produces only good 2 as an a priori restriction. In this case, our method is not applicable to firm 2, as this firm is not able to vary individual prices by changing the output of one good only. However, for firm 1, first-order conditions can still be derived from maximization over prices as in (10).

Remark 1. We have assumed that the profit functions have unique optima in the results above. This, however, is not necessary for the characterization; all we need is that the global maximum of the profit function corresponds to a first-order condition. In practice, one needs to check the second-order conditions to ensure that each first-order condition corresponds to a local maximum, as well as checking for multiple local maxima.

\section{Differentiated Goods: Bertrand vs. Cournot}

As discussed above, Cournot paradigm is preserved by a specific setting of $\mathbf{D}_{\mathbf{p}} \mathbf{Q}^{i}\left(\mathbf{p}^{*}\right)$ while maximization is carried over prices. In this section, the purpose is to clarify the relationship between the Cournot and Bertrand equilibria. However, differentiated goods setting is necessary for well-behaved Bertrand equilibrium.

Suppose that there are $m$ goods, with the demand function given in (5), and $m$ firms where firm $i$ sells only good $i$. For simplicity of exposition, assume that

\footnotetext{
${ }^{6}$ There are three other feasible solutions to (12), however, the second-order conditions are violated at these solutions.
} 
the marginal costs for all goods are constant, denoted by $c_{i}$ for good $i$. Thus, the profit function of firm $i$ is given by,

$$
\Pi^{i}=\left(p_{i}-c_{i}\right) Q^{i} .
$$

If the profit is maximized with respect to price, the first-order conditions are of the form

$$
Q^{i}+\left(p_{i}-c_{i}\right) \mathbf{D}_{p_{i}} Q^{i}=0
$$

where $Q^{i}=f_{i}(\mathbf{p})$ due to the market-clearing condition.

We need the following notation in the statement of the following result: For $\mathbf{x} \in \Re^{m}$, let $\mathbf{x}_{-i} \in \Re^{m-1}$ denote the vector obtained by deleting the $i^{\text {th }}$ row from $\mathbf{x}$. Thus, for firm $i, \mathbf{p}_{-i}$ and $\mathbf{f}_{-i}(\mathbf{p})$ denote the prices and quantities of other firms' goods.

Proposition 4 Suppose that both Bertrand and Cournot equilibria exist where every firm produces a positive amount. Then, the solution to the following system of first-order conditions,

$$
Q^{i}+\left(p_{i}-c_{i}\right) \mathbf{D}_{p_{i}} Q^{i}=0, \quad i=1, \ldots, m,
$$

yields, (i) the Bertrand equilibrium if

$$
\mathbf{D}_{p_{i}} Q^{i}:=\mathbf{D}_{p_{i}} f_{i}(\mathbf{p}),
$$

and (ii) the Cournot equilibrium if

$$
\mathbf{D}_{p_{i}} Q^{i}:=\mathbf{D}_{p_{i}} f_{i}(\mathbf{p})-\mathbf{D}_{\mathbf{p}_{-i}} f_{i}(\mathbf{p})\left(\mathbf{D}_{\mathbf{p}_{-i}} \mathbf{f}_{-i}(\mathbf{p})\right)^{-1} \mathbf{D}_{p_{i}} \mathbf{f}_{-i}(\mathbf{p}) .
$$

Proof. The Bertrand case is true by definition. For the Cournot case, the first-order conditions of the maximization problem w.r.t. quantities are

$$
\left(p_{i}-c_{i}\right)+Q^{i} \mathbf{D}_{Q^{i}} p_{i}=0 .
$$

Let $J=\mathbf{D}_{\mathbf{p}} \mathbf{f}(\mathbf{p})$; the existence of a Cournot equilibrium ensures that $J^{-1}$ exists in the neighborhood of the equilibrium prices. From the implicit function theorem, $\mathbf{D}_{Q^{i}} p_{i}$ is equal to $J_{i i}^{-1}$, i.e., the $i^{t h}$ element of the diagonal of the matrix $J^{-1}$. Let $J_{-i}$ be the matrix obtained by deleting $i^{\text {th }}$ row and $i^{\text {th }}$ column of $J$. Furthermore, let $A_{i}$ and $B_{i}$ be the $i^{t h}$ row and column, respectively, of $J$, with $i^{\text {th }}$ element removed. From matrix algebra, the elements on the diagonal of the inverse of $J$ can be written as,

$$
J_{i i}^{-1}=\left(J_{i i}-A_{i} J_{-i}^{-1} B_{i}\right)^{-1} .
$$

Note that $J_{-i}=\mathbf{D}_{\mathbf{p}_{-i}} \mathbf{f}_{-i}(\mathbf{p}), A_{i}=\mathbf{D}_{\mathbf{p}_{-i}} f_{i}(\mathbf{p})$ and $B_{i}=\mathbf{D}_{p_{i}} \mathbf{f}_{-i}(\mathbf{p})$. Hence, post-multiplying (15) with $\left(J_{i i}-A_{i} J_{-i}^{-1} B_{i}\right)$ for each firm yields the first-order conditions in the proposition.

This is a trivial proposition as the strategic variables in the Cournot game are simply replaced with prices through a change of variables. The interesting 
part of this result is that it affords a price competition interpretation for the Cournot game, reminiscent of Kreps \& Scheinkman (1983). Note that, the total derivative of the quantity with respect to the price can be written as,

$$
\mathbf{D}_{p_{i}} Q^{i}=\mathbf{D}_{p_{i}} f_{i}(\mathbf{p})+\mathbf{D}_{\mathbf{p}_{-i}} f_{i}(\mathbf{p}) \mathbf{D}_{p_{i}} \mathbf{p}_{-i} .
$$

In the Bertrand competition framework, one makes the usual Nash equilibrium assumption that each firm sets its price assuming that its price does not effect the other firms' prices, i.e., $\mathbf{D}_{p_{i}} \mathbf{p}_{-i}=\mathbf{0}$. Thus, one interpretation of the Cournot case is that the firms drop this assumption and foresee that the other firms' prices will be effected through the market clearing conditions when they set their own price.

Quoting from Mas-Colell, Whinston \& Green (1995),

After all, most firms seem to choose their prices, not their quantities. For this reason, many economists have thought that the Cournot model gives the right answer for the wrong reason. (p. 394)

Price competition interpretation of the Cournot game suggests that the reason is, perhaps, not that wrong.

Remark 2. While the price approach to the Cournot equilibrium results in significant computational advantage in the homogenous-good case, no advantage is gained in the differentiated-goods case. From the proof, it is clear that the computation required is equivalent to computing $\mathbf{D}_{Q^{i}} p_{i}$ for all firms.

Remark 3. It is straightforward to generalize Corollary 2 to cases intermediate to homogeneous and differentiated settings, where each firm produces a subset of the goods. The general result is the same as part (ii) of proposition 4 , except that (i) the vectors $\mathbf{p}_{-i}$ and $\mathbf{f}_{-i}$ include only the goods that firm $i$ does not produce, and, (ii) $p_{i}$ and $f_{i}$ are replaced with price and quantity vectors corresponding to the goods that firm $i$ does produce.

\section{References}

Anderson, S. \& Leruth, L. (1993), 'Why firms may prefer not to price discriminate via mixed bundling?', International Journal of Industrial Organization 11, 49-61.

Carbajo, J., Meza, D. \& Seidmann, J. D. (1990), 'A strategic motivation for commodity bundling', The Journal of Industrial Economics 38, 283-296.

Chen, Y. (1997), 'Equilibrium product bundling', Journal of Business 70(1), 85100. 
Kreps, D. \& Scheinkman, J. (1983), 'Quantity precommitment and bertrand competition yield cournot outcomes', Bell Journal of Economics 14, 326337.

Martin, S. (1999), 'Strategic and welfare implications of bundling', Economics Letters 62, 371-376.

Mas-Colell, A., Whinston, M. D. \& Green, J. R. (1995), Microeconomic Theory, Oxford University Press.

Matutes, C. \& Regibeau, P. (1992), 'Compatibility and bundling of complementary goods in a duopoly', The Journal of Industrial Economics 40(1), 3754.

Nalebuff, B. (1999), Bundling as an entry barrier. SSRN working paper, http://papers.ssrn.com/paper.taf?abstract id $=185193$.

Orbay, H. \& Orbay, B. Z. (2006), Imperfect competition and incentives for bundling. Working paper, Sabanci University.

Tirole, J. (1988), The Theory of Industrial Organization, MIT Press, Cambridge, MA.

Vives, X. (1985), 'On the efficiency of bertrand and cournot equilibria with product differentiation', Journal of Economic Theory 36(1), 166-175.

Whinston, M. (1990), 'Tying, foreclosure, and exclusion', American Economic Review 80(1), 837-860. 